\title{
SEHEN UND GESEHEN WERDEN. DIE MACHT DES BlICKES UND DER BLICK ZURÜCK.
}

\author{
Peter Anton Zoettl $1^{1}$
}

Beobachtung während einer partizipativ-audiovisuellen Feldforschung in der Lissabonner Peripherie bilden den Hintergrund für visuell-anthropologische Überlegungen zur „Macht des Blickes“. Der Blick staatlicher Institutionen und der Agenten staatlicher wie gesellschaftlicher Gewalt (wie z. B. der Medien) spiegelt Machtverhältnisse wider und schreibt diese fest. Die Bewohner der unterprivilegierten Stadtteile der portugiesischen Großstädte sind das Objekt eines Blickes, der sie in ihrer Alterität konstituiert und mittels dessen sie der portugiesischen Gesellschaft in ihrer bildhaften Marginalität ,, vorgeführt" werden.

Visuelle Anthropologie, Marginalität, Portugal, Immigration, Drogen

Observations during participatory-audiovisual fieldwork in an immigrant neighbourhood in the outskirts of Lisbon provide the basis for reflections on the state's and society's "power of vision". The eye of state institutions and its agents, and of societal forces (like the media) mirror and establish power relations. The residents of the underprivileged neighbourhoods of the Portuguese metropolises are the object of a dominant eye which constitutes them in their alterity and presents them before Portuguese society in their pictorial marginality.

visual anthropology, marginality, Portugal, immigration, drugs

„Man müsste etwas über die Leute machen, die nachts arbeiten und tagsüber schlafen, und ihre Kinder kaum zu Gesicht bekommen“. „Oder einen Film über die Polizei, über das Verhältnis der Polizei zu den Bewohnern des Viertels“. „Einen Film über den ,Blick der Leute auf das Viertel“, darüber, wie die Leute aus der Nachbarschaft unseren Stadtteil sehen“. „Einen Film über unser Inseldasein, über die Isolierung des Viertels vom Rest der Stadt“. „Das Verhältnis von Eltern und Kindern“. „Die afrikanische Kultur“. „Probleme in der Schule“.

\footnotetext{
${ }^{1}$ Centro em Rede de Investigação em Antropologia (CRIA-IUL), Lissabon. Die dem Artikel zugrundeliegende Forschung wurde von der portugiesischen Fundação para a Ciência e a Tecnologia (FCT) finanziert (Postdoktoranden-Stipendium).
} 
So lauteten die sieben Themenvorschläge der Teilnehmer eines partizipativen Videoworkshops im Lissabonner Stadtteil 6 de Maio, eines kapverdischen Immigrantenviertels am Rande der Stadt. Der Workshop wurde aus verschiedenen Gründen, auf die am Ende näher eingegangen wird, nie zu Ende geführt. Er dient deshalb, zusammen mit einem anderen, im benachbarten Stadtteil Cova da Moura durchgeführten Workshop eher als „Vorwand“ für die folgenden Überlegungen zur „Macht des Blickes“, als dass er als solcher beschrieben und analysiert werden soll. ${ }^{2}$ Die während seines Verlaufs gemachten Beobachtungen bilden den Ausgangspunkt für eine Anthropologie des Sehens, die sich auf die räumlich-visuellen Aspekte der gesellschaftlichen Rangordnung zwischen Zentrum und Peripherie am Beispiel Portugals konzentriert. Während das materielle Ziel des Workshops, dem medialen Blick vom Zentrum in die Peripherie einen lokalen Blick aus dem Stadtteil entgegenzusetzen, nicht erreicht wurde, mag die Reflexion über das „Sehen“ und „Gesehen werden“ nicht nur dem Ethnologen nützlich gewesen sein: Das Erkennen und Zum-Ausdruck-Bringen gesellschaftlicher Zusammenhänge - so, wie es sich in den Äußerungen, Diskussionen, Vorstellungen, Ängsten, Urteilen und Vorurteilen der teilnehmenden Jugendlichen im Laufe des Workshops offenbart hat - ist meist der notwendige erste Schritt, um den eigenen Überzeugungen Gehör und politisches Gewicht zu verleihen (vgl Zoettl 2013).

Die Überzeugung oder, in den Worten des britischen Medien- und Kulturwissenschaftlers John Fiske (2000, 53), das „Wissen“, Objekt eines dominanten Blickes zu sein, ist unter den Bewohnern der Immigrantenviertel der Lissabonner Peripherie weit verbreitet. Das Empfinden, vom Staat und von den Medien beobachtet zu werden, wie auch das Gefühl des Unvermögens, diesem dominanten Blick wirksam die eigene Sicht der Dinge entgegensetzen zu können, ist Ausdruck sozialer Strukturen, die hier vor allem im Hinblick auf ihre visuellanthropologischen und den gesellschaftlichen Raum ordnenden Aspekte erörtert werden sollen. Was lässt sich über die Sichtweisen der Jugendlichen einer ethnisch/kulturell/ökonomischen Minderheit, den auf sie gerichteten Blick von Staat und Gesellschaft und das Verhältnis jener sich kreuzenden Blicke sagen? Sind die von den Jugendlichen

\footnotetext{
${ }^{2}$ Der Workshop im Stadtteil 6 de Maio wurde im Sommer 2011 gemeinsam mit Catarina Vasconcelos und Catarina Laranjeiro in Zusammenarbeit mit dem Centro Social 6 de Maio organisiert; der Workshop in der Cova da Moura in Zusammenarbeit mit der Iniciativa Bairros Críticos Ende 2010. An beiden Workshops nahmen jeweils ca. fünf bis acht jugendliche Anwohner teil, die nach einer eingehenden Diskussion über mögliche Themen, das jeweilige „Skript“ des zu realisierenden Videos und einer kurzen Einführung in Kameratechnik selbstständig ein kurzes Dokumentarvideo produzierten. Währen der Workshop in der Cova da Moura erfolgreich zu Ende geführt wurde (vgl. Zoettl 2012b), ist das Video aus dem 6 de Maio noch immer ,work in progress“ und soll 2013 von einem der Teilnehmer fertiggestellt werden.
} 
des 6 de Maio vorgeschlagenen Filmthemen mehr als nur der Ausdruck der täglichen Nöte und Sorgen der Bewohner eines Immigrantenviertels? Welche Rolle spielen Bewohner, Staat und Medien bei der Konstruktion des öffentlichen Bildes ihres Viertels und anderer, vergleichbarer Stadtteile? ${ }^{3}$

\section{Der Blick auf den Anderen}

Das 6 de Maio ist ein fast ausschließlich „schwarzer“ Stadtteil, der zwar administrativ nicht mehr zur Stadt Lissabon gehört (sondern zum Kreis Amadora), jedoch direkt vor den Toren der Stadt, den Portas von Benfica liegt, eines Wohnviertels der Lissabonner Mittelschicht. Ein Blick auf Google Earth vermittelt einen ersten Eindruck von der „Alterität“ des 6 de Maio, seiner geographischen Andersartigkeit im Vergleich zum städtebaulichen Umfeld der näheren Umgebung. Die verschachtelte Struktur der Häuser ist aus der Satellitenperspektive gut zu erkennen, sie kontrastiert mit der regelmäßigen Anordnung der südlicher gelegenen Wohnblocks, die von Straßen und Grünstreifen umsäumt werden. ${ }^{4}$

Eine Streetview gibt es nicht vom 6 de Maio, der Kamerawagen von Google, Inc. hat an den Portas de Benfica abgedreht und sich wieder dem Stadtzentrum zugewendet, ein Manöver, das das Verhältnis der Lisboetas zu seiner schlechtbeleumdeten Peripherie nachzuzeichnen scheint: Die meisten der zentrumsfernen Stadtteile des Großraums Lissabons genießen einen schlechten Ruf, ein Großteil der afrikanischen Einwanderer des Landes wohnt hier und für viele Portugiesen sind Viertel wie das 6 de Maio oder die benachbarte Cova da Moura „tabu“. Man wendet den Blick von ihnen und bemüht sich um Abstand, wenn nicht gerade Fernsehen und Zeitungen Bilder von Gewalt und Drogen ins Wohnzimmer tragen, die aus sicherer Distanz betrachtet werden können.

\footnotetext{
${ }^{3}$ Auf eine medienethnologische Auseinandersetzung mit der „Macht des Blickes“ wird hier weitgehend verzichtet. Ein Überblick über medienethnologischen Forschungen zu dem hier behandelten Themenkomplex findet sich z. B. in Cottle (2000) oder Kraidy (2007). Insbesondere Stuart Hall hat sich bereits Ende der 70er Jahre mit dem Thema der medialen Aufarbeitung des Themas Minderheiten/Gewalt gewidmet (Hall 1978). Downing and Husband $(2005,25)$ beklagen in diesem Zusammenhang den hauptsächlich textorientierten Fokus der meisten Untersuchungen.

${ }^{4} \mathrm{Http} / / /$ maps.google.de/maps?ll=38.750477,-9.213415\&spn=0.002631,0.005681\&t=h\&z=18. BEI URL's BITTE IMMER DAS DATUM DES ZUGRIFFS ANGEBEN., besucht am 26.3.2013
} 
Auch die Isolation des Viertels ist bereits von oben auszumachen, das 6 de Maio beschränkt sich auf ein vielleicht 150 Meter im Quadrat großes Gelände, das im Westen, Norden und Osten von Fabrikgebäuden umschlossen ist, im Süden von der Estrada Militar, die hier die Grenze zum eigentlichen Lissabonner Stadtgebiet zieht. Lissabon ist in den letzen fünfundzwanzig Jahren merklich gewachsen, der Beitritt Portugals zur Europäische Wirtschaftsgemeinschaft 1986 und die Jahre um die Weltausstellung 1998 haben der Mittelschicht eine stetig wachsende Kaufkraft beschert und Portugal von einem Land der Emigranten (eine Million Portugiesen wohnen allein in Frankreich) zum Einwanderungsland gemacht. ${ }^{5} \mathrm{Zu}$ den seit dem Ende der Kolonialkriege (1961-1974) zugereisten Afrikanern (hauptsächlich aus Kapverden, Angola und Guinea-Bissau) haben sich im letzten Jahrzehnt Brasilianer und Ukrainer gesellt die, wie ihre afrikanischen Kollegen, zumeist in der Bauindustrie oder im Restaurantgewerbe ihr Auskommen finden. Die Peripherie der Stadt ist so nach und nach, vor allem entlang der ins Inland getriebenen Bahnlinien und auf der gegenüberliegenden Seite des Rio Tejo, zu einem dichtbesiedelten, breiten Gürtel herangewachsen, der die Zahl der Einwohner, die sich als Lissabonner fühlen und bezeichnen, in den letzten Jahrzehnten vervielfacht hat. Fast drei Million Menschen wohnen heute in der Área Metropolitana de Lisboa, ein Viertel der portugiesischen Gesamtbevölkerung.

Es sind jedoch nicht geographische Aspekte, auf die sich die Teilnehmer des Videoworkshops beziehen, wenn sie von ihrem „Inseldasein“ und der „Isolierung des Viertels“ sprechen. Das 6 de Maio liegt kaum fünf Gehminuten vom Vorortbahnhof Damaia entfernt, von dem aus man in genau acht Minuten mit der Regionalbahn im Herzen der Stadt, am Rossio, ist. Es geht ihnen vielmehr um die empfundene, erlebte und gelebte Isolation; um das Gefühl, nicht dazuzugehören, „mitten in der Stadt“ zu wohnen, aber kein Teil dieser zu sein.

Das 6 de Maio ist eine eigene, kleine Welt, die sich nicht nur aus der Satellitenperspektive von ihrer Umgebung unterscheidet: Die eng nebeneinander wie übereinander konstruierten Häuschen der Bewohner haben ein enges Labyrinth von Gassen entstehen lassen, das an man-

\footnotetext{
${ }^{5}$ Mit der Verschärfung der Wirtschaftskrise in Portugal und dem sich verschiebenden globalen ökonomischen Gleichgewicht hat sich diese Bewegung in den letzten Jahren wieder umgekehrt: Viele der Anfang des 20. Jahrhunderts zugewanderten Immigranten verlassen das Land, in der Hoffnung, in ihren nun wirtschaftlich aufstrebenden Heimatländern Fuß zu fassen (Brasilien, Angola); auch die Zahl der emigrierenden Portugiesen hat wieder deutlich zugenommen. Während etwa in 2008 noch ca. 16.000 Menschen von außerhalb der EU nach Portugal einwanderten, verzeichnet die Statistik für 2010 nur noch gut 5.500 Einwanderer; die Zahl der Auswanderer betrug 2008 ca. 20.000 und in 2010 über 23.700 (INE 2012, 118f.).
} 
chen Stellen kaum einen Meter breit ist. Der Eindruck der Abgeschlossenheit wird durch die deutliche schwarz-weiß Trennung von Bewohnern, Anwohnern und Gelegenheitsbesuchern noch verstärkt. Südlich der Estrada Militar beginnt das „weiße“ Benfica, dem das 6 de Maio wie eine schwarze Trutzburg gegenüberzustehen scheint. Die Jugendlichen, die an den Kreuzungspunkten der Gassen mit der Estrada Militar zusammenstehen, vermitteln Außenstehenden den Eindruck, die Zugänge ins Innere des Viertels würden „,bewacht“. Der rege Austausch zwischen „schwarz“ und „weiß“, der sich von der gegenüberliegenden Straßenseite aus beobachten lässt, entpuppt sich bei näherem Hinsehen jedoch als das Kommen und Gehen der im Centro Social 6 de Maio tätigen Sozialarbeiter und einer stattlichen Zahl (weißer) Drogenkonsumenten, die, wie Baltazar ${ }^{6}$, einer der Workshop-Teilnehmer es ausdrückt, auf dem „,mercado“ (Markt) des 6 de Maio „einkaufen gehen“.

De facto überschattet der Drogenhandel sowohl das Alltagsleben im 6 de Maio als auch die Beziehung seiner Bewohner zur unmittelbaren Nachbarschaft und zur portugiesischen Gesamtgesellschaft. Das öffentliche Bild der Bewohner des Viertels (so wie das anderer illegaler Siedlungen am Stadtrand) fußt größtenteils auf einer, von den Medien in Umlauf gebrachten, „Ikonographie“ des Drogenhandels und Drogenkonsums. Die Vorstellung vom „Inseldasein“ des 6 de Maio steht somit im Wechselspiel zu dem ebenfalls vorgeschlagenen Filmsujet des „Blick[s] der Leute auf das Viertel“: Die Ablehnung, die die Bewohner des 6 de Maio - aufgrund der im öffentlichen Diskurs vorherrschenden Assoziation ihres Viertel mit Drogen und Gewalt - erfahren und zu erfahren meinen, ist Ursache einer gefühlten Isolation, die oft zur Selbstisolation führt.

Die vermeintliche Gewaltbereitschaft der Jugendlichen der Peripherie spiegelt sich, im öffentlichen Diskurs und in den Medien, hauptsächlich in den häufigen Einsätzen der Polizei in Vierteln wie dem 6 de Maio. Auseinandersetzungen zwischen der PSP (Polícia de Segurança Pública) und den Bewohnern des Viertels sind hier, wie auch in anderen ,problematischen“ Stadtteilen Lissabons, beinahe an der Tagesordnung. Die Teilnehmer des Workshops bringen so immer wieder die Sprache auf „die Polizei“, wenn es ihnen darum geht, den Blick Außenstehender auf das Viertel und seinen ,schlechten Ruf“ zu beschreiben. Nur wenige Wochen vor unserem ersten Treffen waren bei einem Einsatz der PSP mehrere Bewohner verletzt wor-

\footnotetext{
${ }^{6}$ Namen geändert.
} 
den, unter ihnen ein Säugling. Das Auftreten der Polizei wird von den Jugendlichen als generell vorurteilshaft, diskriminierend und gewaltprovozierend beschrieben.

Einen Film über das Verhältnis der Bewohner zur Polizei will man jedoch nicht machen: Zu groß ist die Sorge, den Unwillen der Agenten der staatlichen Exekutive auf sich zu ziehen, deren Verhalten von den Bewohnern als schwer berechenbar eingeschätzt wird. Nicht wenige der Jugendlichen im 6 de Maio (und insbesondere in der Cova da Moura) haben bereits unliebsame Erfahrungen mit den Beamten der für sie zuständigen Esquadras (Polizeistationen) gemacht, und berichten von nicht nur sporadischen physischen Aggressionen. ${ }^{7}$ So einigt man sich schließlich darauf, zunächst die Frage nach dem „Inseldasein“ des Viertels filmisch umzusetzen, und vielleicht ein Interview mit der Leiterin der örtlichen Polizeiwache zu führen, die, so Baltazar, offenbar „,keine gute Meinung von den Bewohnern des 6 de Maio hat““.

Sich filmisch über den „Anderen“ Gedanken zu machen ist eine neue Erfahrung für die Teilnehmer des Workshops. Sie sind daran gewöhnt, das Ziel und nicht der Ausgangspunkt eines kollektiven Blickens zu sein; daran, von den Medien befragt und gefilmt zu werden, und tags darauf sich selbst und ,ihresgleichen“ in Presse und Fernsehen wiederzufinden. In der Berichterstattung der portugiesischen Medien über Drogen, Gewalt und Polizeieinsätze in den afrikanischen Vierteln der Lissabonner Peripherie haben die Bewohner meist ganz buchstäblich das Nach-Sehen: Es bleibt ihnen nichts übrig, als abzuwarten, wie ihre Ausführungen von den Journalisten innerhalb der Narrationen der medialen Berichterstattung (um-)gedeutet werden. Ihr photographisches Abbild in der Zeitung oder im Fernsehen macht die Bewohner zum bildhaften Objekt einer journalistischen Wertung, die in der Regel nicht mit ihrem Selbstbild in Einklang steht.

\footnotetext{
${ }^{7}$ Vgl. hierzu z. B. die „Concluding observations“ des Human Rights Committee der Vereinten Nationen (Item 10): „The Committee continues to be concerned about reports of excessive use of force and ill-treatment by law enforcement officials and members of the security forces" (UNHRC 2012: 3). Im Bericht des Europäischen Komitees zur Verhütung von Folter und unmenschlicher oder erniedrigender Behandlung oder Strafe ist von „numerous allegations of illtreatment by law enforcement officials“ die Rede: „The allegations received during the 2008 visit mainly concerned slaps, punches and blows with various objects such as batons and telephone books; allegations of verbal intimidation and of a specific threat made with a firearm were also received." (CPT 2009: 12). Der Bericht des Portugalbesuchs der Delegation von Anfang 2012 lag bei Drucklegung noch nicht vor.
} 


\section{Der öffentliche Blick}

Während der „Blick der Leute“ aus der Nachbarschaft seine Entsprechung in eigenen Beobachtungen, Interpretationen und auch Vorurteilen gegenüber „den Portugiesen“ findet, ist der mediale Blick - der aufgrund seines öffentlichen Charakters den Blick der portugiesischen Gesellschaft in ihrer Gesamtheit zu repräsentieren scheint - ein einseitiger. Er kann zwar kritisiert und kommentiert werden (was auch reichlich geschieht), aber nur schwer zurückgeworfen, da den auf ihn bezogenen Erwiderungen in der Regel eben jene Öffentlichkeit fehlt, die den Blick der Medien so zwingend erscheinen lässt. Dem Blick der Medien ist kaum etwas entgegensetzen, da er weder von einem Einzelnen ausgeht (dem man sich entgegenstellen könnte), noch sich an einen Einzelnen richtet. Sein Ziel sind nicht die Individuen und Gruppen, die von ihm in Bild und Ton erfasst werden, sondern jene, an die der mediale Blick weitergereicht (,übertragen“) wird: die fiktive gesellschaftliche Allgemeinheit, der die journalistischen Berichterstattung im Akt des medialen Blickens die „Anderen“, auf die geblickt wird, gegenübergestellt - und somit in ihrer vermeintlichen Alterität erst konstituiert und sichtbar macht.

Wie in den meisten afrikanischen Vierteln der Stadt haben die Bewohner des 6 de Maio keine gute Meinung von „den Journalisten“. Man wirft ihnen vor, nur für negative Schlagzeilen zu sorgen und die, durchaus vorhandenen, Probleme mit Gewalt und Drogen grob zu überzeichnen. Tatsächlich haben sich Viertel wie das 6 de Maio und insbesondere die benachbarte Cova da Moura in Portugal als mediales Symbol für städtische Problemzonen etabliert. Die Bewohner und ihr Viertel sind so in der portugiesischen Öffentlichkeit zum Sinn-Bild für „Störungen der öffentlichen Ordnung“, ,zunehmende Kriminalität““ oder ein „Gefühl der Unsicherheit“ geworden.

Die unterprivilegierten Stadtteile der Peripherie repräsentieren solche, im öffentlichen Diskurs vor allem zu Wahlkampfzeiten beliebte, Schlagworte nicht nur „exemplarisch“ (d. h. als vom Einzelfall abgeleitete Argumente zur Verteidigung bestimmter allgemeiner politischer oder privater Ansichten), sondern auch „bildhaft“ im Zuge einer piktographischen Generalisierung, bei der das konkrete Abbild des Anderen für eine Vielzahl diffus umgrenzter Attribute, Situationen, Meinungen und Gefühle steht. Diese bildschriftliche Generalisierung lässt sich z. B. in Fernsehbeiträgen beobachten, in denen Aufnahmen ,generischer“ Afro-Portugiesen als Hin- 
tergrundbilder für Reportagen über Kriminalität in „,schwarzen“ Vierteln Verwendung finden, für die es (aufgrund eines fehlenden konkreten Bezugs oder der Abwesenheit eines Kameramanns zum Zeitpunkt des Geschehen) keine tatsächlich referentiellen Bilder gibt. Von den Betroffenen wird dieser Missbrauch des Bildes von Menschen einer bestimmten ethnischen Herkunft deutlich wahrgenommen und als Beleg für die Voreingenommenheit der portugiesischen Gesellschaft gegenüber der afrikanischstämmigen Minderheit zitiert. Ein Bewohner der Cova da Moura etwa beklagte sich mir gegenüber über die Verwendung von Aufnahmen aus dem Fernseharchiv, auf denen er zu sehen ist, die jedoch tatsächlich in keinerlei zeitlichem oder inhaltlichem Zusammenhang zu der von ihm kritisierten Reportage über „Gewalt“ im Stadtteil standen.

\section{Der Blick aus der Ferne}

Die Bildsprache von Presse und Fernsehen folgt meist einfachen Mustern, die aus der Distanziertheit des von ihr praktizierten Blickes folgen. Die ungleiche Machtbalance zwischen Beobachter und Beobachteten, die dem nicht zurückwerfbaren medialen Blick zugrundeliegt, schafft auch physische Distanz. So wird das 6 de Maio in den Medien meist in seiner Außenansicht abgebildet, da ein „Eindringen“ des Kamerateams in das unübersichtliche Labyrinth der Gassen aufgrund des wenig vertrauensvollen Verhältnisses von Medien und Bewohnern auf Missfallen stoßen würde. Die mediale Außenansicht wiederum verstärkt den bildhaften Eindruck einer „schwarzen Trutzburg“, eines rechtsfreien Raums, in den, wie es im öffentlichen Diskurs heißt, selbst die Polizei nur mit Spezialeinheiten vorrücken könne. Die erwähnten, an den Kreuzungspunkten von Estrada Militar und den in das Viertel führenden Gassen „herumlungernden“ Jugendlichen wiederum werden, aus der gleichen Kameraperspektive, zum bildhaften Ausdruck unsteten Lebenswandels (sie gehen anscheinend keiner geregelten Beschäftigung nach), Kriminalität (beziehen ihr Einkommen also wohl aus illegalen Aktivitäten) und Rechtlosigkeit (all das geschieht am helllichten Tag).

Der mediale Kamerablick ist von Susan Sontag (1973 [2005], 43) als „,voyeuristisch“ denunziert worden: Sein Ziel, so Sontag, sei nicht die (An-)Teilnahme am Abgebildeten, sondern das Einfangen bildlicher faits divers, mit denen die soziale Realität auf die Vorstellungswelt der - vom oberflächlichen Blick der Kamera erhaschten - Bilder reduziert werde. Die Beliebigkeit der Aneinanderreihung unterschiedlichster Ereignisse (,ein Strom unterschiedsloser 
Bilder, die das jeweils vorangehende für nichtig erklären“, ebd., 20) homogenisiere die Lebenswelten, die sich vor den Augen der Kamera abspielen: „enttäuschte Hoffnungen, die Launen der Jugend, Kolonialkriege und Wintersportarten, alles ähnelt einander““ (ebd., 13).

Doch der mediale Blick aus der Distanz macht nicht nur „gleich“, er vereint das Abgebildete auch visuell in Klassen, in denen die Unverwechselbarkeit und Individualität des Gefilmten hinter die Wiederholung sich ähnelnder Bildern zurücktreten. Die Bilder afro-portugiesischer Jugendlicher, die am Rande der Stadt augenscheinlich in den Tag hineinleben, sind nicht nur „,sinn-los“, insofern sie, für sich selbst genommen, nicht nach Ursachen oder Zusammenhängen fragen (,stumm und nackt, platt und dumpf“, wie Dubois es ausdrückt ${ }^{8}$ ). Sie identifizieren den einzelnen Bewohner auch bildlich mit all den anderen „schwarzen“ Bewohnern der Vorstädte und bildhaft - im journalistischen Zusammenspiel von Bild und Text - mit den mit der Lissabonner Peripherie konnotierten Schlagworten und Aussagen. So repräsentiert am Ende jeder einzelne Bewohner des 6 de Maio oder der Cova da Moura im Diskurs der Medien weniger sich selbst, als sein Viertel und die zentrumsfernen Stadtteile an sich - und hat in der Folge im öffentlichen Diskurs für das, was sich in ,seinem“Viertel, tatsächlich oder mutmaßlich, abspielt den (medialen) Kopf hinzuhalten. ${ }^{9}$ Für die Teilnehmer des Workshops, die die Macht des medialen Blicks aus eigener Anschauung kennen, ist der Blick der Medien mehr als nur das Zerrbild einer von ökonomischen und politischen Interessen beeinflussten Nachrichtenindustrie. Der Blick der Medien deckt sich für sie mit dem Blick des „weißen“ Nachbarn, in dem sich die vermuteten Vorurteile der portugiesischen Gesellschaft zu spiegeln scheinen.

Der Blick der Medien ist gleichzeitig unidirektional (er lässt sich nicht „,zurückwerfen“) und selektiv: Die Autorität der Medien bezieht sich neben der Form der Darstellung auch auf die Auswahl der Themen. Der mediale Blick zeigt, was Macher und Zuschauer (aus welchen Gründen auch immer) für sehenswert halten. ${ }^{10}$ Der Selektivität der Medien den eigenen Standpunkt entgegenstellen zu können, ist den Teilnehmern des Workshops deshalb ein wichtiges Anliegen: Während es Baltazar vor allem darum geht, ,endlich die Dinge zeigen zu

\footnotetext{
${ }^{8}$ Dubois 1998, 87.

${ }^{9}$ Vgl. hierzu Halls (1978, 3f) Beobachtungen bzgl. der Genese des Begriffs „,mugger“ in Großbritannien und der auf äußerlich sichtbaren Merkmalen beruhenden Klassifizierung vermeintlicher Gewalttäter. Fiske wiederum verweist auf den homogenisierenden $(2000,61)$ und kategorisierenden $(1996,249 f)$ Charakter der polizeilichen und kommerziellen Videoüberwachung in den USA.

${ }^{10}$ Zur Selektivität der Medien (,framing“) vgl. auch Downing and Husband (2005, 36).
} 
können, die wir zeigen möchten“, will Arlindo (in dem Workshop in der Cova da Moura) der Öffentlichkeit „ein anderes Bild von unserem Viertel““ vermitteln ${ }^{11}$. Von den Jugendlichen des 6 de Maio wird die filmische Auseinandersetzung mit „afrikanischer Kultur“ oder „Leuten, die nachts arbeiten und tagsüber schlafen“ (zwei der ursprünglichen Themenvorschläge) insofern als Chance verstanden, eine andere Facette des 6 de Maio sichtbar zu machen - Menschen, die arbeiten, wie andere auch und die etwas vorzuweisen haben, auf das sie stolz sein können. Neben dem Verlangen, die Berichterstattung der Medien „richtig zu stellen“ (Baltazar) und der eigenen Narration (z. B. über den jüngsten Polizeieinsatz) Gehör zu verschaffen, steht der Wunsch, den öffentlichen Blick dorthin zu lenken, wo Normalität wie Spezifität des eigenen Viertels und der eigenen Kultur in Erscheinung treten.

\section{Der gerichtete Blick}

In der Art und der Richtung des Blickes, der zwischen Gesellschaft und Individuum, zwischen gesellschaftlichen Gruppen und Individuen (derselben oder anderer Gruppen) hin und hergeht, spiegelt sich ihr Verhältnis. Der Blick, mit dem die Alterität des Anderen ,abgeschätzt“ wird, ist nicht nur Ausdruck interpersoneller Sympathie oder Abneigung (wie etwa in den sich kreuzenden, ,zwischen-menschlichen“ Blicken), sondern oft auch Ausdruck und Bedingung gesellschaftlicher Machtverhältnisse. Der Blick der Medien etwa, der sich auf die Bewohner des 6 de Maio richtet, von diesen jedoch nicht wirksam (da nicht öffentlich) beantwortet werden kann, offenbart die untergeordnete Position der peripheren Lissabonner Minderheiten im gesellschaftlichen Diskurs ebenso, wie er ihr Unvermögen, auf den öffentlichen Diskurs Einfluss zu nehmen (mit-)begründet.

Die Einseitigkeit des Kamerablicks ist die Konsequenz bestimmter Formen medialen Berichtens, die den Objektstatus des Abgebildeten visuell festschreiben. Der Blick der Medien bahnt sich seinen Weg durch das Objektiv der Fernsehkamera zum Individuum, welches aber nicht der Kamera (dem Kameramann) in die Augen sieht, sondern dem Journalisten. Der Blick des Abgebildeten begegnet somit nicht den Augen der Zuschauer, die ihn daher auf den Fernsehschirmen nicht als handelndes Subjekt, als sondern behandeltes sujet erleben.

\footnotetext{
${ }^{11}$ Teilnehmerinterview von Catarina Laranjeiro und Catarina Vasconcelos (2010).
} 
Wie Dubois (1998) bemerkt, dient im Spielfilm - der sich, scheinbar im Gegensatz zur Fernsehreportage, dem Möglichen und nicht dem Tatsächlichen widmet - die Abwesenheit des Kamerablicks dazu, die Fiktion der Realität der inszenierten Handlung für den Betrachter aufrechtzuerhalten und so zu verhindern, „dass die geschlossene Welt der Fiktion aufbricht und im Bild die Präsenz des Operators markiert wird [...], wo doch die traditionelle Fiktion gerade auf der Absenz oder auf der Auslöschung dessen beruht, was ihren Ursprung ausmacht [...]“ (ebd., 179). Das Fernsehen hingegen, das sich dem Realismus der Nachrichtenberichterstattung verschrieben hat, beruft sich als gesellschaftliche Kraft gerade auf seine NichtSubjektivität, d. h. den Anspruch (erfüllt oder nicht), eine vom Individuellen befreite Sicht der Dinge zu vermitteln und An-Sichten auszustrahlen, die über die persönliche Meinung des berichtenden Journalisten hinausgehen. Die postulierte Objektivität der Nachrichtenmedien beruht auf dem Anspruch, nicht die eigene Narration zu verbreiten, sondern „Fakten“ in die Wohnstube zu übertragen - so, als würde der Blick des Kameramanns nur an die Zuschauer weitergereicht.

Der Blick der Fernsehkamera, der auf dem Bildschirm mit dem Blick des Zuschauers verschmilzt, muss sich deshalb, um seine „Objektivität“ formalästhetisch zu unterstreichen, auf den Standpunkt des omnipräsenten und allwissenden Erzählers zurückziehen. Er darf dem sujet nicht visuell begegnen (das tut der im Auftrag der Medien tätige Journalist), sondern es nur aus der Ferne betrachten. Der Blick des sujet in die Kamera würde dieses zum Subjekt machen, zum Gegenüber von Kameramann und Zuschauer, und damit dem eigentlichen Subjekt (dem Journalisten und der fiktiv von ihm vertretenen „Öffentlichkeit“) die Zügel aus der Hand nehmen, mit denen das Fernsehen die Gefahr, die Subjektivität seiner Narration augenscheinlich werden zu lassen, zu bändigen sucht. Das in die Kamera blickende sujet würde zudem, indem sein Blick den Weg zum Zuschauer zurückwandert, diesen auf sich selbst ,zurückverweisen“ und so in seinem Voyeurismus und, wie Dubois es ausdrückt, seiner „Dumm-

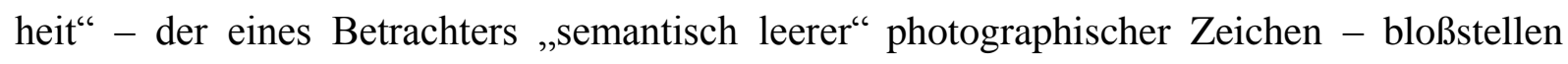
(ebd., 179, 87).

\section{Der machtvolle Blick: Zur-Schau-Stellen, Verbergen, Ausforschen}

Der Blick der Medien ist fast immer ein ein-seitiger. Im 6 de Maio und in der Cova da Moura erlebt und empfindet man diese Einseitigkeit und beklagt sich über die „Negativität“" von 
Presse und Fernsehen in, zunächst überraschendem, Einklang mit den Politikern. Auch diese fordern häufig, im krisengeschüttelten Portugal doch ,auch mal das Positive zu zeigen“, nicht immer ,alles kaputtzureden“. Zu den illegalen Siedlungen am Rande der Stadt jedoch hat die Politik ein gespaltenes Verhältnis: Zu Wahlkampfzeiten besuchen die Kandidaten für das Amt des Präsidenten, Premierministers oder Bürgermeisters gerne die Cova da Moura oder andere „,verrufene“ Bezirke, die der breiten Öffentlichkeit aus den Medien ein Begriff sind. Gegenüber potentiellen Wählern (mit portugiesischem Pass) aus Kapverden, Angola oder Guinea bekunden die Kandidaten so ihre Bereitschaft zur Integration, den „richtigen“ Portugiesen gegenüber hingegen ihren Willen, die sozialen Probleme des Landes „,anzupacken“. Zwar ist Lissabon im internationalen und europäischen Vergleich eine sehr sichere $\operatorname{Stadt}^{12}$, doch hat auch hier das subjektive Empfinden der Unsicherheit, insbesondere im Hinblick auf eine augenscheinlich höhere Kriminalitätsrate unter Immigranten, in den letzten Jahren stetig zugenommen.

Der Staat hat auf dieses (von ihm selbst instrumentalisierte) Empfinden ,reagiert“ und die Strafgesetze verschärft. Im portugiesischen Strafgesetzbuch von 1995 etwa finden sich eine Reihe von Änderungen gegenüber dem von 1982, die vor allem als „schwer“ klassifizierte Delikte deutlich härter bestrafen - eine Gesetzesänderung, die mit Portugals Öffnung und seiner Entwicklung vom Auswanderungs- zum Einwanderungsland in Verbindung gebracht wird (vgl. Cunha 2002, 47ff; Cunha 2010). Cunha verweist in diesem Zusammenhang auf die Popularität von Schlagworten wie ,, alarme social “ („öffentliches Aufsehen“) und „Immigration“ innerhalb der Strafrechtsdiskussion. Das „öffentliche Aufsehen“ - das sich in den (halböffentlichen) Gesprächen in Cafés und tascas (Kneipen) und der medialen Berichterstattung Gehör verschafft - ist hierbei gleichzeitig Ausdruck und Wegbereiter des politische Diskurses zur inneren Sicherheit: Der alarme social und das abstrakte Gefühl großstädtischer Unsicherheit dienen der Politik als willkommenes Argument für die Ausweitung des staatlichen Gewaltmonopols; andererseits betont der politische Diskurs seinerseits regelmäßig die „faktische“ Notwendigkeit einer stärkeren staatlichen Präsenz in den „städtischen Problemzonen“ (,zonas urbanas sensiveis“). So wurde beispielsweise die Anschaffung von sechs Panzerfahr-

\footnotetext{
${ }^{12}$ Im Eurostat Bericht 58/2010 Crime and Criminal Justice belegt Lissabon mit 0,64 Tötungsdelikten pro 100.000 Einwohnern einen der unteren Plätze (im Vergleich: Berlin 1,31 oder London 2,17); der Durchschnittswert für die Jahre 2007-2009 (Eurostat „Statistics in focus“, 6/2012) beträgt für Lissabon 0,48 (Berlin: 1,93; London: 1,92).
} 
zeugen der Beschussklasse B6/BR6 ${ }^{13}$ für den NATO-Gipfel in Lissabon im November 2010 zunächst mit dem erhöhten Gefährdungspotential eines internationalen Treffens gerechtfertigt, schließlich jedoch (da die Fahrzeugen erst nach dem Ende des Gipfels in Lissabon eintrafen) mit der innerstädtischen Bedrohungslage begründet. Die gepanzerten Fahrzeuge, so der Sprecher der PSP, sollten „die polizeilichen Einsatzkräfte und andere Personen bei etwaigen Zwischenfällen schützen, zu denen es in städtischen Problemzonen kommen könnte" ${ }^{\prime 14}$. Der erste „Einsatz“ eines der Fahrzeuge fand im Juni 2011 statt - im Stadtteil 6 de Maio, wenige Tage nach dem erwähnten Zusammenstoß zwischen Polizei und Bewohnern, um „,eventuellen Auseinandersetzungen im Zusammenhang mit den Unruhen die Stirn zu bieten““15.

Da die engen Gassen des 6 de Maio die Zirkulation von Kraftfahrzeugen nicht erlauben, beschränkte sich die Jungfernfahrt auf das „,demonstrative“ Abstellen des Panzerwagens vor der nahegelegenen Polizeiwache von Damaia. Die Zurschaustellung des martialischen Polizeigefährts veranschaulicht eine der Spielarten des „,machtvollen“ staatlichen Umgangs mit Fragen der Sichtbarkeit, die sich analytisch drei (sich überlappenden) Handlungsfeldern zuordnen ließen: das Zur-Schau-Stellen, das Verbergen und das Ausforschen. Mit der Zurschaustellung staatlicher Macht hat man es dort zu tun, wo (wie vor der Polizeistation von Damaia) der Durchsetzung des staatlichen Gewaltmonopols dienende Apparaturen und Institutionen bewusst öffentlich sichtbar gemacht werden (Militärparaden, Polizeiaufgebote, Grenzanlagen, Regierungsgebäude, etc.).

Foucault $(1994,15)$ referiert, wie noch im 18. Jahrhundert der Staat seine Macht auch durch das Zurschaustellen von Bürgern (am Pranger) verteidigt, die mit ihrem als „Verbrechen“ klassifizierten Verhalten das staatliche Gewaltmonopol zu schwächen drohen. Die ostentativen Spielarten der Zurschaustellung von Macht (der Pranger, die Marter, etc.), so Foucault, werden vom Staat jedoch schließlich abgeschafft, weil sie sich als ineffektiv erweisen. Wer sich der Staatsgewalt widersetzt - etwa indem er raubt oder mordet oder aber „landstreicht“ (also nicht arbeitet und somit nicht zur Akkumulation von Reichtum beiträgt, der zum Erhalt

\footnotetext{
${ }^{13}$ Schutz gegen "Angriffe durch organisierte Kriminalität oder Terroristen mittels Gewehren oder Militärwaffen; begrenzt gegen Sprengungen“ bis Kaliber 7,62 (http://www.power-trax.de/produkte/armored-vehicles/armoredvehicles.htm, besucht am 17.10.2011)

${ }^{14}$ Paulo Flor zit. nach der Wochenzeitung Sol vom 17.10.2011, http://sol.sapo.pt/inicio/Politica/Interior.aspx?content_id=5810 (besucht am 17-10-11).

${ }^{15}$ Blog der Unidade Especial de Polícia (Spezialeinheiten der PSP),

http://pormaioriaderazao.blogspot.com/2011/06/psp-estreia-blindado-para-travar.html, besucht am 20.10.2011
} 
der staatlichen Gewaltmonopols unabdingbar ist) - wird nun, anstatt öffentlich bestraft, weggesperrt.

Mit der systematischen Einrichtung von Gefängnissen im Europa des Anfangs des 19. Jahrhunderts wird die Behandlung von Straftätern vom Justizapparat auf den Strafvollzug übertragen und dem Blick der Öffentlichkeit entzogen. Nur die Gefängnismauern selbst bleiben für jedermann sichtbar und gesellen sich - als steingewordener Ausdruck staatlicher Macht - zu dem Ensemble von Palästen, Regierungsgebäuden und Polizeistationen. Im Gefängnis vereinen sich so die Funktion des Verbergens der freiheitsentziehenden staatlichen Gewalt und der Zurschaustellung der staatlichen Sanktionsmacht.

In der Stadt Lissabon gibt es zwei Gefängnisse, die dem Vollzug von Freiheitsstrafen dienen, das Estabelecimento Prisional de Lisboa und das Estabelecimento Prisional de Monsanto. Ersteres wurde im Zuge der Abschaffung der Todesstrafe 1867 als Hauptgefängnis der Stadt konzipiert und 1885 fertiggestellt. Seine Konstruktion folgte ausdrücklich dem architektonischen Projekt von Jeremy Benthams Panopticon. Wie sein Vorbild ist auch das Lissabonner Gefängnis Ausdruck einer neuen Methodik des Staates im Umgang mit dem Rechtsbrecher: „Wir bestrafen zwar, doch wollen wir damit eine Heilung erreichen“ (Foucault 1994, 33). Doch die panoptische Strafanstalt verkörpert nicht nur Ideale wie Disziplinierung und Resozialisierung, sie unterstützt auch, mit architektonischen Mitteln, den Machtanspruch und die Effektivität des staatlichen Blickes. Die vollständige „Kontrolle der Gefangenen“ etwa wird im Estabelecimento Prisional de Lisboa durch die radiale Struktur seiner „sechs Flügel, mit jeweils vier Stockwerken“ garantiert, „die an einem Punkt zusammenlaufen, von dem aus der zentrale Korridor eines jeden Flügels überwacht wird“. ${ }^{16}$ Der Gefängnisaufseher, der in dem durch die zusammenlaufenden Flügel gebildeten zentralen Ort seinen Dienst tut, kann so die Gefängnisflure überblicken, ohne selbst von den in den Zellen weilenden reclusos (Strafgefangenen) gesehen zu werden, ganz im Sinne von Foucaults Interpretation des Benthamschen Entwurfs:

\footnotetext{
${ }^{16}$ Webseite der Direcção-Geral dos Serviços Prisionais (Generaldirektion der Strafvollzugsbehörde), „Estabelecimentos Prisionais - Estabelecimento Prisional de Lisboa“, besucht am 22.10.2011 (http://www.dgsp.mj.pt/).
} 
„Jeder Käfig ist ein kleines Theater, in dem jeder Akteur allein ist, vollkommen individualisiert und ständig sichtbar. [...] Das Prinzip des Kerkers wird umgekehrt, genauer gesagt: von seinen drei Funktionen - einsperren, verdunkeln und verbergen - wird nur die erste aufrechterhalten [...]. Jeder ist an seinem Platz sicher in einer Zelle eingesperrt, wo er dem Blick des Aufsehers ausgesetzt ist; aber die seitlichen Mauern hindern ihn daran, mit seinen Gefährten in Kontakt zu treten. [...] Er wird gesehen, ohne selber zu sehen; er ist Objekt einer Information, niemals Subjekt in einer Kommunikation.“ (Foucault 1994, 256f.)

In der funktionalen Logik der Architektur des Panopticons und seiner Lissabonner Variante lassen sich Elemente einer „Grammatik des Blickens“ identifizieren, die auch im modernen Rechtsstaat Anwendung finden. So ist auch im Lissabonner Gefängnis der Blick des Staates auf die Eingeschlossenen ein exklusiver Blick, kein öffentlicher. Auch die „moderne“ portugiesische Öffentlichkeit weiß nur über die Tatsache der Verurteilung der Gefangenen Bescheid, nicht jedoch darüber, was sich genau im Inneren des Gefängnisses abspielt. Während zu Zeiten der Marter jedermann mit ansehen konnte (und sollte) welche Qualen der Machthaber dem Rechtsbrecher zuteil werden ließ, ist der Vollzug der Gefängnisstrafe fast immer den Blicken nicht-staatlicher Augen entzogen. Weder der (individuelle) Bürger, noch die gesellschaftlichen Öffentlichkeit (z. B. die Presse) haben gemeinhin die Möglichkeit in Augenschein zu nehmen, auf welche Weise das staatliche Gewaltmonopol im Gefängnis vollzogen wird. $^{17}$

\section{Der Blick auf das Viertel}

Das öffentliche Zurschaustellen von Bürgern, deren Lebensweise nicht im Einklang mit den gesellschaftlichen Normen steht, wird im modernen Staat de facto hauptsächlich von den Medien übernommen. ${ }^{18}$ Die Augen der Medien ersetzen die Augen des Staates, die sich aus der Öffentlichkeit zurückgezogen haben und nun im Verborgenen arbeiten, hinter den Mauern der Gefängnisse etwa oder im Zuge einer immer unauffälligeren wie umfassenderen Überwachung der Staatsbürger mittels elektronischer Sammlung und Auswertung von Daten und Bil-

\footnotetext{
${ }^{17}$ Einem Bericht des European Committee for the Prevention of Torture and Inhuman or Degrading Treatment or Punishment (CPT 2009, 45) zufolge starben im Jahr 2007 insgesamt 76 Strafgefangene in portugiesischen Gefängnissen (davon 10 Selbsttötungen). Derselbe Bericht referiert: "[T]he CPT's delegation did receive a certain number of allegations of physical ill-treatment of prisoners by custodial staff [...]. These allegations concerned punches, kicks and blows with batons, after the prisoners concerned had been brought under control, and in certain instances the persons apparently required medical treatment as a result." (ebd., 24).

${ }^{18}$ Vgl. hierzu auch Fiske (1996, 260): „Neither Orwell nor Foucault, the founding theorists of panopticism, envisaged the possibility that the media might make panoptic power public and thus both share its pleasures and expose its operation."
} 
dern. Es ist jedoch meist nicht der Straftäter, der in den Medien der Öffentlichkeit „,vorgeführt“ wird (dieser genießt in den meisten europäischen Ländern ein „Recht am eigenen Bild“ und kann sich auf die Unschuldsvermutung berufen). Vorgeführt wird den Zusehern vielmehr das photographische Abbild des potentiellen Delinquenten, die „einzelne Manifestation des globalen Phänomens der Kriminalität“ (Foucault 1994, 325). Gerade weil er sich „noch“ nichts zu Schulden hat kommen lassen, muss jener es sich gefallen lassen, ins Blickfeld der Fernsehkameras gerückt und der Fernsehöffentlichkeit präsentiert zu werden.

Der namenlose Delinquent muss der Öffentlichkeit viel gefährlicher erscheinen als der individuelle Straftäter, der ja schon gefasst oder zumindest als solcher erkannt und somit für die Agenten staatlicher Macht greifbar ist. Bereits im beginnenden 19. Jahrhundert, so Foucault (1994, 369), kommt den Medien eine wichtige Rolle bei der Konstitution des Delinquenten als „überall gegenwärtig[em] und überall gefährlich[em]“ Störer der öffentlichen Ordnung zu. Tag für Tag, so Foucault, berichte die Presse „,von dem inneren Kampf gegen einen antlitzlosen Feind“ und stelle ,in diesem Krieg die tägliche Alarm- oder Siegesmeldung dar“ (ebd.).

Auch der Blick der Medien auf die Bewohner des 6 de Maio oder der Cova da Moura ist solch ein weitschweifender, tagtäglicher Blick auf einen gesichtslosen Feind, dem die Kamera nicht in die Augen sieht, sondern aus der Ferne ablichtet: Die Individualität der Gefilmten ist in der Berichterstattung aus der Peripherie Lissabons gerade deshalb irrelevant, weil in ihr meist nicht von konkreten und tatsächlichen, sondern von abstrakten und potentiellen Gesetzesübertretungen gesprochen wird. Die auf den Gassen „herumlungernden“ Jugendlichen sind keine Täter, sondern - im Kontext der Medienberichterstattung - potentielle Delinquenten. Nicht der (mutmaßliche) Joint, der „am helllichten Tage“ und „vor aller Augen“ geraucht wird, ist die eigentliche Nachricht, die der Öffentlichkeit mit den vom medialen Blick eingefangenen Bildern überbracht werden soll, sondern die mit der vorstädtischen Jugendkultur assoziierten Gefahren für die öffentliche Ordnung und die Sicherheit des ,gemeinen“ Staatsbürgers.

Im öffentlichen Diskurs der Moderne hat sich der Handel mit den vom Staat als Drogen klassifizierten Substanzen längst als vermeintliche Ursache eines ganzen Komplexes von Gefahren für die „,̈ffentliche Ordnung“ etabliert. Die „Figur des Drogenhändlers“, schreibt Manue- 
la da Cunha (2002, 325), „scheint sich - so wie die des Landstreichers im 19. Jahrhundert, zu dessen symbolischen Gegenstück sie geworden ist - als der Prototyp gesellschaftlicher Bedrohungen und gefährlicher Verhältnisse etabliert zu haben“. Die Verschärfung der Strafgesetzgebung für Drogendelikte, die präventive, auf bestimmte Zonen der Stadt konzentrierte Polizeiarbeit und die prozessuale Praxis der Zusammenlegung von Strafverfahren mehrerer Angeklagter (als „Bande“ oder „kriminelle Vereinigung“) haben, so Cunha, in Portugal dazu beigetragen, dass das „Gefängnis den Stadtteil in sich aufgenommen hat" (ebd., 200). Die Insassen des von ihr untersuchten Frauengefängnisses von Tires etwa

\footnotetext{
„entstammen alle denselben Vierteln - Barackensiedlungen, einzelnen Baracken oder vorfabrizierten Häusern, Umsiedlungsvierteln oder suburbanen Sozialwohnungssiedlungen [...]. 89\% der Gefängnisinsassinnen aus dem Großraum Lissabon kommen aus solchen Vierteln, ein ähnlicher Anteil wie im Großraum Porto (86\%).“(Cunha 2002, 76)
}

Die Schuld der aus den Barrackensiedlungen und Sozialwohnungsbauten der Peripherie stammenden Verdächtigen, Verhafteten, Untersuchungsgefangenen und Verurteilten ist, so wie die von Foucaults Delinquenten, über-individuell. Cunha zeichnet die „kollektivierenden Mechanismen" nach, die dazu geführt haben, dass sich die peripheren Stadtteile ins Gefängnis hinein ,erstrecken“ (ebd., 26, 195) und verweist auf die zunehmende Auflösung der „symbolischen Grenze“ zwischen „Drinnen“ und „Draußen“ seit den 80er Jahren. Ein Gefängnisaufenthalt, so Cunha, sei für viele Bewohner der Peripherie der portugiesischen Großstädte zum „banalen und normalen“" Teil der Vita geworden (ebd., 199).

Die Blicke von Staat und Medien auf die Peripherie gleichen sich in ihrer Ausrichtung, insofern sie deren Bewohner nicht differenzieren, sondern ent-individualisieren. Die ,generischen“ Afro-Portugiesen, die in den Medien bildlich mit dem diffusen Drogenkomplex assoziiert werden, werden auch vom Staat nicht als Individuen, sondern als Gruppe (und Klasse) ins Auge gefasst. Während jedoch z. B. Hall (1978), Wacquant (2002, 56) oder Fiske (1996; 2000) für den britischen und nordamerikanischen Kontext eine klare ethnische „Präferenz“ des Gefängnissystems ausmachen, betont Cunha die primär räumliche Selektivität des polizeilichen Blickes: 
„Im Gegensatz zu Straftaten, die nur durch die Anzeige des Opfers bekannt werden, beruht die Aufdeckung von Drogendelikten auf vorbeugenden Ermittlungen der Polizei. Im Rahmen der Selektivität dieses vorbeugenden Ins-Auge-Fassens ziehen bestimmte soziale und räumliche Gruppen kontinuierlich die polizeiliche Aufmerksamkeit auf sich. Innerhalb eines solchen Rasters der Verdachtsentstehung ist der persönliche sozioökonomische oder ethnisch-,,rassische“ Status von geringerer Bedeutung als der Status des, als Einheit betrachteten, Wohnviertels.“ (Cunha 2002, 309f.)

Cunhas Beobachtung zeigt, dass hinter dem selektiven Blick des Staates auf seine Bürger mehr steckt als die „einfache“ Diskrimination ethnischer Minderheiten. Der Blick des Staates ist Teil einer gesellschaftlichen Raumordnung des Sehens, in dessen Zentrum die Institutionen politischer Macht und der institutionalisierten Öffentlichkeit (die Medien) stehen und an dessen Rand sich jene gesellschaftliche Gruppen befinden, die aufgrund ihrer potentiellen Gefahr oder Nutzlosigkeit für das Funktionieren von Wirtschaft und Verwaltung ins Visier der Agenten des Staates genommen werden.

\section{Lokalität und Sichtbarkeit}

Außerhalb des Gefängnisses, in den unübersichtlichen Gassen des 6 de Maio, hat es die staatliche Macht bedeutend schwerer, einen Blick auf die Bürger zu werfen, ohne selbst gesehen zu werden. Der Polizist in Uniform ist als Agent staatlichen Sehens nur bedingt nützlich, da er nicht nur Erfüllungsgehilfe staatlicher Macht ist, sondern diese auch repräsentiert. Als sichtbarer Ausdruck des staatlichen Gewaltmonopols ist er, wie das vor der Polizeistation geparkte Panzerfahrzeug, beinahe ein „Relikt“ der performativen Aspekte staatlicher Macht - der Agent einer staatlichen Exekutive, die nicht mehr exekutiert oder martert. Die öffentlich ausgetragenen Auseinandersetzungen zwischen der PSP und den Jugendlichen des 6 de Maio oder der Cova da Moura stören die Raumordnung des Sehens eher, als dass sie ihr dienten. Staatstragend sind sie nur insoweit, als sie der Öffentlichkeit die Gefährlichkeit der Bewohner der Peripherie ,vor Augen führen“ und die Gesamtgesellschaft so auf Maßnahmen vorbereiten, mittels derer die Raumordnung des Sehens künftig effektiver, d. h. diskreter, umgesetzt werden soll.

Mit ihrem System verwinkelter Gassen und der verschachtelten Ansammlung von Häusern und Barracken sind die meisten Stadtteile der Lissabonner Peripherie das genaue Gegenteil eines panoptischen „Theaters“ ständig sichtbarer Akteure. In vielen Stadtteilen der Lissabon- 
ner Peripherie treten die Agenten der staatlichen Exekutive deshalb nicht nur in Uniform, sondern auch à paisana (in Zivil) oder als homens de confiança (V-Männer) auf. Die „Vertrauensleute“ des Staates sind u. a. gesetzlich ermächtigt, Drogen zu erwerben oder „kontrolliert weiterzugeben“ (Cunha 2002, 51). Ihre eigentliche Funktion ist es jedoch, Informationen über die Modalitäten und Akteure des Drogenhandels oder sonstiger Straftaten zu sammeln. Ihre Arbeit dient nicht nur dazu, den uniformierten Agenten zuzuarbeiten (z. B. indem sie zur Fahndung ausgeschriebenen Verdächtige ausfindig machen), sondern soll auch zur präventiven Überwachung eines Stadtteils, der für Außenstehenden nur schwer einsehbar ist, beitragen. Mit seiner unauffälligen Präsenz bringt der homem de confiança Licht ins Dunkel der Peripherie und stattet die staatliche Macht so „mit einer ununterbrochenen, erschöpfenden, allgegenwärtigen Überwachung [aus], die imstande ist, alles sichtbar zu machen, sich selber aber unsichtbar“" (Foucault 1994, 275).

Neben der Architektur ist es auch ihre „Lokalität“, die Stadteile wie das 6 de Maio für den Staat (und die Medien) so schwer durchschaubar machen. Eine ehemalige Bewohnerin des 6 de Maio etwa betonte in einem von den Teilnehmern des Workshops gefilmten Interview ihre anhaltende Verbundenheit mit dem Viertel, in dem, trotz aller Probleme, ein ausgeprägtes Gemeinschaftsgefühl vorherrsche. Das 6 de Maio sei

„eine Art zu leben, eine Gemeinschaft. Dort draußen gibt es das, was wir hier im Viertel leben, nicht. Wenn jemand krank wird, bemühen sich alle, ihn zu besuchen und zu sehen, ob alles in Ordnung ist. Jemand der mit finanziellen Probleme zu kämpfen hat und irgendwann nicht mehr weiß, was er seinem Kind zu Essen geben soll, dem bezahlt der Nachbar das Brot. Wir sind eine Gemeinschaft hier.“

Auch in der benachbarten Cova da Moura hat sich, trotz des schlechten Rufs, den der Stadtteil in der portugiesischen Öffentlichkeit genießt, im Laufe der Jahre (hauptsächlich dank der Arbeit einiger lokaler Vereine) ein Gefühl von communitas herausgebildet. Die Bewohner anderer, zwischenzeitlich abgerissener ${ }^{19}$, illegaler Siedlungen, die in weit vom Zentrum entfernte Sozialwohnungskomplexe umgesiedelt wurden, hingegen beklagen (neben den weiten Wegen, die ihre neuen Wohnstätten von ihren Arbeitsplätzen trennen und der prekären Infra-

\footnotetext{
${ }^{19}$ Z. B. die Fontainhas (2005) oder die Azinhaga dos Besouros (2006), deren Bewohner größtenteils Wohnungen in den (mit öffentlichen Verkehrsmitteln schwer zu erreichenden) Sozialbauten im Casal da Mira zugewiesen wurden. Seit Mitte 2012 forciert die Kreisverwaltung von Amadora den Abriss des Stadtteils Santa Filomena. Auch das 6 de Maio soll abgerissen werde, während für die Cova da Moura verschiedene architektonische Restrukturierungpläne erarbeitet wurden (vgl. Malheiros 2006).
} 
struktur) vor allem das Fehlen von solidarischen nachbarlichen Beziehungen. Nachbarschaftliche „Lokalität“ jedoch steht, wie Arjun Appadurai bemerkt hat, nicht unbedingt im Einklang mit den Ordnungsbestrebungen des Staates:

\footnotetext{
"Neighbourhoods, as social formations, represent anxieties for the nation-state, since they usually contain large or residual spaces where the techniques of nationhood (birth-control, linguistic uniformity, economic discipline, communications efficiency and political loyalty) are likely to be either weak or contested. [....] For the project of the nation-state, neighbourhoods represent a perennial source of entropy and slippage. They need to be policed almost as thoroughly as borders.” (Appadurai 1995, 218)
}

Der Abriss illegaler Siedlungen der zentrumsnahen Peripherie und die Umsiedlung in Sozialbauten der zentrumsfernen Peripherie bricht gewachsene Nachbarschaftsstrukturen auf und vereinzelt die Bewohner. Während z. B. das Straßenbild in der Cova da Moura von Menschen geprägt ist, die sich, im Gespräch mit Nachbarn und Bekannten, vor ihren Häusern aufhalten, gibt es in den oft für mehrerer hundert Familien konzipierten Komplexen des portugiesischen sozialen Wohnungsbaus nur wenige Orte, die den nachbarlichen Kontakt förderten. Den etwa 5.000 Bewohnern der Cova da Moura stehen innerhalb ihres Viertels beinahe 150 Geschäfte (Cafés, Friseure, Gemüseläden, etc.) zu Verfügung. ${ }^{20}$ In den meisten bairros sociais (Sozialwohnungsviertel) hingegen gibt es in der Regel nur einige wenige Cafés; die eigentlich vorgesehenen Ladengeschäfte stehen aus Angst vor Vandalismus oft leer.

Die seit den 90er Jahren in Lissabon und Porto durchgeführten Umsiedlungen ${ }^{21}$ arbeiten so einer sozialen Raumordnung zu, die der Disziplinierung der Bürger der Peripherie (im Sinne Foucaults) dienlich ist, indem sie die auf, wie Appadurai es ausdrückt (1995, 219), ,verhältnismäßig stabilen Verbindungen, verhältnismäßig bewussten und miteinander geteilten Geschichten und gemeinsam durchschrittenen, lesbaren Räumen und Plätzen“ aufbauenden „neighbourhoods-life-worlds“ durch homogenisierte und von Individualität wie Gruppengefühl befreite Orte ersetzen. In den Siedlungen des sozialen Wohnungsbaus unterstützt die Architektur die Ordnungsbestrebungen des Staates. Wie die Gefangenen in Benthams Panopticon oder im Lissabonner Zentralgefängnis sind die dort ansässigen Mieter der untersten Einkommensklassen einerseits gut sichtbar und andererseits von einander (so wie von den besserverdienenden Bewohnern des Stadtzentrums) isoliert. Zum einen wirkt die offene Anla-

\footnotetext{
${ }^{20}$ Malheiros $(2006,91)$.

${ }^{21}$ In Umsetzung des Programa Especial de Realojamento (PER), Decreto-Lei nº.163/93.
} 
ge der Gebäude (keine verwinkelten Gassen, sondern für jedermann einsehbare Plätze und Straßen) Handlungen entgegen, die normalerweise nur im Verborgenen stattfinden; zum anderen erschwert die funktionalistische Architektur den nachbarlichen Kontakt und das Entstehen von „Lokalität“, indem sie die Bewohner durch die Abwesenheit sozialer Treffpunkte und die, Anonymität provozierende, Aneinanderreihung von Hochhausapartments effektiv voneinander fernhält.

Die bairros sociais gleichen so Foucaults „lebenden Tableaus“, die aus den „unübersichtlichen, unnützen und gefährlichen Mengen“ der in Barrackensiedlungen hausenden Individuen „geordnete Vielheiten“ machen $(1994,190)$. Die geographische Selbstordnung der Städte (in Lissabon wohnten viele der ersten afrikanischen Immigranten der 70er Jahre noch im zentralen Stadtteil São Bento, nicht weit vom Parlamentsgebäude) ist nicht nur Ausdruck und Folge der Einkommensdifferenzierung der Konsumgesellschaft (vgl. Baudrillard 1970 [2008]), sie ist auch Teil einer Raumordnung, die der Aufrechterhaltung der staatlichen Ordnung zuarbeitet. Die unübersichtliche Ungeordnetheit von Vierteln wie dem 6 de Maio begünstigt das Entstehen von lokalem Gemeinsinn im gleichen Maße wie sie die Überwachung der Anwohner erschwert. Sie sind dem staatlichen Blick nur zugänglich, indem dieser sich selbst undercover der Unordnung unterordnet. Die „Delinquenz“, die als Rechtfertigung der (selbst am Rande der Legalität operierenden) verhüllten Tätigkeit des staatlichen Blickes dient, ist generisch, eine Delinquenz des Raumes: Nicht das Handeln des einzelnen Bürgers ist es, das den Verdacht begründet, sondern der Ort seine Aufenthalts. Der Drogenhandel, der sich im öffentlichen Diskurs wie ein Schleier über die Peripherie Lissabons zu legen scheint, ist Ursache, Folge und Symbol dieser Delinquenz, die, wie Manuela da Cunha beschreibt, Gefängnis und Stadtteil immer enger verschränkt:

\footnotetext{
„Die massenhaften Karrieren vom Viertel ins Gefängnis gehen hauptsächlich auf den Drogenhandel zurück, zu einem guten Teil als Folge der kollektivierenden Mechanismen, mit denen dieser bekämpft wird und mittels derer die ,gefährlichen Klassen` räumlich abgesondert werden, aufs neue vereint mit den wirtschaftlich schwächsten Schichten der,Arbeiterklasse‘. Hier wie anderswo produziert die Welt der Drogen einen Sicherheitsdiskurs, der weniger Individuen als ganze Gesellschaftsschichten zum Ziel hat [...].“(Cunha 2002, 327)
} 


\section{Die Macht des Blickes und der Blick zurück}

Der „Blick zurück“ der Teilnehmer des Workshops machte auf halber Strecke halt: Mit den beginnenden Sommerferien verließen einige die Stadt, andere arbeiteten als Betreuer auf den traditionellen portugiesischen Feriencamps für Schulkinder oder schafften es nicht mehr, Schule, Ausbildung und die Betreuung der eigenen Kinder mit außerplanmäßigen ,kulturellen“ Aktivitäten zu vereinbaren. Der Film über den „Blick auf das Viertel“ blieb bis auf weiteres unvollendet. Wie Shirley White (2003) in ihrem Reader „Participatory video: images that transform and empower" referiert, steht der Teilnahme am gesellschaftlichen Diskurs oft die Notwendigkeit im Wege, wirtschaftlich zu überleben. Partizipation, so White, ,erfordert persönliche Opfer“, die von Menschen aus marginalisierten Bevölkerungsschichten oft nur schwer erbracht werden können, da diese oft „vollständig mit dem täglichen Kampf um das Wohlergehen und der Ernährung ihrer Familie beschäftigt sind“ (ebd., 45).

Auch die Idee, sich filmisch gegen die polizeilichen Übergriffe auf die Bewohner des Viertels zur Wehr zu setzen, wurde sowohl im 6 de Maio als auch in der Cova da Moura schließlich wieder aufgegeben. Sie müssten, so drückten es zwei der Jugendlichen aus, am Ende „früher oder später den Kopf dafür hinhalten“, im wörtlichen Sinne. Wie auch bei anderen von mir organisierten Videoworkshops (vgl. z. B. Zoettl 2011; 2012a; 2012b; 2013) lag den angehenden Filmemachern der Lissabonner Peripherie die positive Darstellung ihrer Kultur und Lebenswelt näher als eine öffentlich geäußerte Kritik an ihren Lebensumständen - eine Beobachtung, die von anderen Sozialwissenschaftlern wie -arbeitern bestätigt wird (vgl. Carelli 2006, 1; Corrêa 2006a, 12; Flores 2005, 10; Guidi 2003, 259).

Der Spielraum, der dem nicht-dominanten Gebrauch von Medien innerhalb der etablierten gesellschaftlichen Machtordnung des Sehens zugestanden wird, hat seine Grenzen. Auch wenn eine zunehmende Zahl von Bewohner der Lissabonner Peripherie inzwischen versucht, das oft unkontrollierte Handeln der Staatsgewalt in ihren Vierteln (möglichst unauffällig) mit Mobiltelefonen zu filmen, sind es nicht nur mangelhafte Technik und fehlendes Know-how, die den Nutzen von ,, videolow“ (Fiske 1996) im portugiesischen Kontext einschränken. Das geringe Interesse der portugiesischen Bevölkerung an den Problemen von Immigranten, die gerade in Zeiten der Krise - nur sehr begrenzt als Bereicherung der heimischen Kultur wahrgenommen werden, sowie die überkommenen Strukturen in Justiz und Verwaltung entziehen 
dem mit der Kamera aufgezeichnete Blick zurück schnell seine gesellschaftliche Relevanz. Für Videotechnik als „guerrilla weapon“, wie sie von Fiske (1996, 227) für den USamerikanischen Kontext gepriesen wird, fehlen in Portugal (das bis Mitte der Siebzigerjahre auf die Wiedereinführung demokratische Strukturen warten musste) schlicht die soziokulturellen Bedingungen.

Dennoch, auch im 6 de Maio machten die ersten Erfahrungen der Teilnehmer mit dem aktiven Gebrauch des Mediums Film deutlich, wie wichtig es ist, gegen die Gewohnheit der öffentlichen „Sprachlosigkeit“ der Bewohner anzugehen. Baltazar etwa, der sich zu Beginn des Workshops noch standhaft weigerte, bei den Interviews mit Anwohnern und Portugiesen aus der Nachbarschaft das Mikrofon in die Hand zu nehmen (aus der Überzeugung heraus, nicht gut genug die Landessprache zu beherrschen), erwies sich schließlich bei dem ersten von ihm geführten Interview als ebenso souveräner wie sensibler Gesprächspartner. Das Bewusstsein „etwas zu sagen zu haben“ ist für viele marginalisierte Gesellschaftsgruppen keineswegs selbstverständlich, und die Erfahrung, dass die eigene Meinung und die eigene Weltsicht auch für andere interessant sein können, ein erster Schritt in Richtung „Empowerment“.

Der filmische „Blick zurück“, als Teil eines Prozesses von Bewusstseinsbildung und Reflektion über die eigene Identität und die eigene Stellung in der Gesamtgesellschaft, versucht so Mechanismen entgegenzuwirken, die der brasilianische Pädagoge Paulo Freire (ganz ähnlich wie Bourdieu Jahre später) als die ,reelle Gewalt“ gesellschaftlicher Strukturen bezeichnet hat, die die Menschen daran hinderten „,mehr zu sein“ (Freire 1968 [2005], 47). Auch der Blick der Medien, der „machtvolle“ Blick des Staates und die gesellschaftliche Raumordnung des Sehens sind Teil einer symbolischen Gewalt mit der, in der Sprache Freires, die „,kulturellen Invasoren“ den Unterschichten ihr eigenes Bild von der Welt aufdrängen und so deren Kreativität und die eigenständige Entwicklung einer Sicht der Dinge unterdrücken (ebd., 173). Das Bestreben, den Blick vom Zentrum in die Peripherie ,zurückzuwerfen“ kann jedoch nur dort Erfolg haben, wo der Blick von „unten“ nach „oben“ das Ergebnis einer tatsächlichen Bewusstseinsbildung über die Bedingungen und Folgen ungleicher Machtverhältnisse ist. Die Herausforderung für den Sozialwissenschaftler, so Freire, liegt „nicht darin, den Leuten unsere Weltsicht" zu vermitteln“, sondern in dem Bestreben, die Menschen, die scheinbar den 
Gegenstand der wissenschaftlichen Betrachtung darstellen, als handelnde Subjekte einer gemeinsamen Reflektion zu begreifen. ${ }^{22}$

\section{Literatur}

Appadurai, Arjun (1995): The production of locality. In: Richard Fardon (Hg.):

Counterworks. Managing the Diversity of Knowledge. London, S. 208-229.

Barthes, Roland (1972): Mythologies. New York.

Baudrillard, Jean (1970 [2008]): A sociedade de consumo [La société de consommation]. Lisboa.

Carelli, Vincent (2006): Moi, un Indien. Catálogo da «Mostra Vídeo nas Aldeias: Um Olhar Indígena». Rio de Janeiro.

Corrêa, Mari (2006a): Conversa a cinco. Catálogo da «Mostra Vídeo nas Aldeias: Um Olhar Indígena». Rio de Janeiro.

Cottle, Simon (2000): Media research and ethnic minorities: mapping the field. In: Simon Cottle (Hg.): Ethnic minorities and the media: changing cultural boundaries. Buckingham, S. 1-30.

CPT (2009): Report to the Portuguese Government on the visit to Portugal carried out by the European Committee for the Prevention of Torture and Inhuman or Degrading Treatment or Punishment (CPT) from 14 to 25 January 2008. Strasbourg.

Cunha, Manuela Ivone da (2002): Entre o bairro e a prisão: tráfico e trajectos. Lisboa.

- (2010): Race, Crime and Criminal Justice in Portugal. In: Anita Kalunta-Crumpton (Hg.): Race, crime and criminal justice: international perspectives . New York, S. 144-161.

Downing, John / Husband Charles (2005): Representing race: racisms, ethnicities and media. London.

Dubois, Philippe (1998): Der fotografische Akt. Versuch über ein theoretisches Dispositiv [L'acte photographique]. Amsterdam.

Fiske, John (1996): Media matters: race and gender in U.S. politics. Minneapolis.

- (2000): White watch. In: Simon Cottle (Hg.): Ethnic minorities and the media: changing cultural boundaries. Buckingham, S. 50-66.

Flores, Carlos (2005): Video indígena y antropología compartida: Una experiencia colaborativa con videastas Maya-Q'Eqchi' de Guatemala. In: Liminar. Estudios Sociales y Humanísticos III(2), S. 7-20.

Foucault, Michel (1994): Überwachen und Strafen. Die Geburt des Gefängnisses. Frankfurt a. M..

Freire, Paulo (1968 [2005]): Pedagogia do oprimido. Rio de Janeiro.

Guidi, Padma (2003): Guatemalan Mayan women and participatory visual media. In: Shirley White (Hg.): Participatory video: images that transform and empower. New Delhi, S. 252-270.

Hall, Stuart (1978): Policing the crisis: mugging, the state, and law and order. London.

INE (2012): Estatísticas Demográficas 2010. Lisboa.

Kraidy, Marwan M. u. a. (2007): Review Essay: Race, ethnicity and global communication studies. In: Global Media and Communication 3(3), S. 371-383.

Malheiros, Jorge et al. (2006): Operação Cova da Moura. Iniciativa Operações De Qualificação E Inserção Urbana Em Bairros Críticos. Volume I - Diagnóstico para a Intervenção Sócio-Territorial. Lisboa.

\footnotetext{
${ }^{22}$ Freire 1968 [2005]: 100, 114 (meine Hervorhebungen).
} 
Sontag, Susan (1973 [2008]): On photography. London.

UNHRC (2012): Concluding observations on the fourth periodic report of Portugal. No place.

Wacquant, Loïc (2002): From slavery to mass incarceration. Rethinking the ,racequestion' in the US. In: New Left Review 13, S. 41-60.

White, Shirley (Hg.) (2003): Participatory video: images that transform and empower. New Delhi.

Zoettl, Peter Anton (2011): Imagens do nordeste, nordeste das imagens. Notas de campo sobre o poder de nomeação, o 'vídeo participativo' e a imagem quilombola. In: Cadernos do Leme 3(1), S. 71-85.

- (2012a): Creating images, creating identity: participatory filmmaking as an anthropological praxis. In: Journal des anthropologues 130-131, S. 53-78.

- (2012b): Participatory Video as a means of reflection and self-reflection about the image and identity of reemerging indigenous groups in north-eastern Brazil. In: Anthropology in Action 19(2), S. 17-26.

- (2013): Images of culture: Participatory video, identity and empowerment. In: International Journal of Cultural Studies 16(2), S. 209-224. 